\title{
Utilisation du modèle de classe inversée pour l'introduction des Séries de Fourier en première année universitaire
}

\section{Using flipped classroom to introduce Fourier series for first year university students}

\author{
Faten Khalloufi-Mouha ${ }^{1 *}$ \\ ${ }^{1}$ Université de Carthage. Faculté des Sciences de Bizerte, TUNISIE
}

\begin{abstract}
Résumé. Dans ce papier, nous décrivons la mise en place d'une expérience de classe inversée pour l'introduction de la notion de série de Fourier à des étudiants de première année universitaire en sciences de l'informatique de la faculté des sciences de Bizerte en Tunisie. Dans l'objectif d'explorer l'impact de ce dispositif pédagogique sur l'apprentissage des mathématiques et en se plaçant dans le cadre de la théorie commognitive, nous avons cherché à étudier l'appropriation par les étudiants, des routines identifiées dans les capsules vidéos qui introduisent le contenu mathématique visé. Sur le plan pédagogique, les premiers résultats font apparaitre une hétérogénéité importante chez les étudiants qui a amené l'enseignante que nous sommes à mettre en place une méthode hybride qui consiste à introduire une séance de synthèse qui a suivi la visualisation des capsules vidéos. Sur le plan didactique, nous avons étudié l'individualisation par les étudiants des trois types de routines associées aux séries de Fourier : les routines de traitement graphique, les routines de traitement calculatoire et les routines de justification et de preuve.
\end{abstract}

\begin{abstract}
In this paper we investigate the impact of the implementation of the flipped classroom approach on the mathematical learning of the Fourier series. The experiment was conducted with a group of first-year computer sciences students from a Tunisian faculty of sciences. Using the commognitive theory, we focus on the students' individualization of routines introduced in the video lectures providing the mathematical content. The pedagogical analysis shows a significant heterogeneity among the students which led the teacher to move to a blended learning by introducing a summary session following the out-of-class part of the flipped classroom method. On the didactic level, we studied the individualization by the
\end{abstract}

* Corresponding author: faten.khalloufi@,fsb.u-carthage.tn 
students of three types of routines associated to the Fourier series: graphics routines, calculation routines and substantiation routines.

\section{Introduction et problématique}

La notion de série de Fourier occupe une place importante dans l'enseignement de l'Analyse au niveau universitaire et admet plusieurs applications dans les différentes sciences et domaines comme la physique, la biologie, les sciences de l'informatique et les différents domaines des ingénieurs. Cependant, très peu de travaux de recherche ont abordé l'enseignement et l'apprentissage de cette notion $[1,2]$. La notion de série de Fourier est une notion ayant un caractère formalisateur susceptible d'être à l'origine de beaucoup de difficultés chez les étudiants qui n'arrivent pas à lui donner un sens et se limitent à une application rituelle des techniques proposées dans le cours. L'étude de l'enseignement des séries de Fourier fait apparaitre dans les cours proposés, une introduction formelle suivie par un passage rapide vers les techniques de calcul des coefficients de Fourier ainsi que la donnée des théorèmes déterminant les conditions de convergence (théorème de Dirichlet et égalité de Parseval). Cet enseignement traditionnel, proposé sous forme de cours magistraux suivi de séances de travaux dirigées, privilégie l'aspect formel ainsi que l'aspect algébrique et calculatoire. Le recours aux aspects visuels et graphiques reste très limité et supposé comme allant de soi chez les étudiants. Le problème est plus accentué pour les étudiants appartenant à des disciplines non-mathématiques pour qui les mathématiques sont supposées être un outil pour résoudre des problèmes liés à leur discipline de spécialité. Pour ces étudiants les séries de Fourier sont introduites d'une façon très formelle qui ne leur permet pas de voir son utilité et de comprendre l'idée d'interpréter certains signaux comme résultant de la superposition d'une infinité de signaux sinusoïdaux de fréquences différentes. Ainsi, ces étudiants se trouvent incapables de construire une signification qui permet de donner un sens aux séries de Fourier. Ce qui engendre généralement, un manque d'intérêt chez les étudiants lors des séances de cours et une importance au niveau des difficultés lors des phases de résolution des activités proposées.

Dans l'objectif de proposer un enseignement efficace de la notion de série de Fourier, les médiateurs visuels graphiques peuvent jouer un rôle très important afin de permettre aux étudiants de donner un sens à cette notion. Cette idée est appuyée par les différents travaux ayant abordé la notion de série en général avec les cas des séries numériques, des séries entières et des séries de Fourier. Ces recherches s'accordent sur le fait que l'enseignement classique utilise très peu de représentations graphiques ce qui peut influencer l'appropriation de cette notion par les étudiants et ne leur permet pas de lui donner une signification et ainsi de réduire son apprentissage à l'acquisition d'un ensemble de savoirs faires qui se traduisent par l'application des théorèmes de convergence. Alcock et Simpson [3] soulignent l'importance du « raisonnement visuel » pour aider les étudiants à établir des liens entre les représentations formelles et visuelles de ce concept. L'idée est également adoptée par Code et Sierra (2007 Cité dans [4]) qui considèrent que les représentations graphiques (notamment les représentations dynamiques) aident les étudiants à visualiser et comprendre le processus en jeu lors de l'étude de la convergence des séries. Ils ont ainsi fait appel dans leur travail à un environnement informatique et ils ont mis en évidence que les représentations graphiques générées dans cet environnement permettent à certains étudiants de mieux comprendre les processus de convergence et d'étendre leurs représentations personnelles à propos de la notion de série. Reis et al. [1] ont souligné que malgré l'importance des séries de Fourier dans la formation des ingénieurs d'électricité, l'enseignement proposé se limite à proposer un savoir-faire permettant aux étudiants de résoudre les activités proposées. Ces auteurs considèrent cet apprentissage comme insuffisant puisque les futurs ingénieurs ont besoins de 
comprendre le concept afin de pouvoir l'utiliser dans les problèmes relatifs à leur discipline (traitement du signal, théorie de l'information, traitement de l'image...) Pour cela ils ont proposé une application Android téléchargeable gratuitement sur les appareils mobiles des étudiants, permettant de faire des approximations des signaux, en utilisant le développement en série de Fourier et cela en se basant sur des représentations graphiques du signal et des approximations en série de Fourier.

Dans ce travail, en se plaçant dans la lignée des travaux précédents, nous adoptons l'hypothèse de l'importance des médiateurs visuels graphiques pour donner un sens aux séries de Fourier et identifier son utilité et son éventuelle utilisation pour la résolution de problèmes. Cependant, nous soulignons la difficulté de réaliser cet objectif dans un enseignement classique vu la difficulté de faire les représentations graphiques des approximations de la fonction par les sommes partielles de la Série de Fourier ainsi que la difficulté de matérialiser et de visualiser la variation selon le rang de la somme partielle. Afin d'éviter les contraintes relatives à un enseignement classique, nous avons choisi de faire appel à une méthode alternative basée sur l'utilisation du dispositif de la classe inversée. L'objectif étant d'offrir aux étudiants un enseignement innovant et actif leur permettant un apprentissage flexible et motivant lors de l'introduction de la notion de séries de Fourier et qui permet de relier ces séries à leurs applications dans le domaine des sciences de l'informatique : la théorie du signal, la transmission des sons et des images, ...

Dans ce travail, nous étudions l'impact de l'implémentation du dispositive de la classe inversée sur l'apprentissage des séries de Fourier par des étudiants de première année des sciences de l'informatique. En se plaçant dans le cadre théorique de l'approche commognitive, nos analyses portent sur l'individualisation par les étudiants de trois catégories de routines : les routines de traitement graphique, les routines de traitement calculatoire et les routines de justification et de preuve. Dans ce papier, nous focalisons notre attention essentiellement sur la première catégorie.

\section{Le dispositif de la classe inversée}

Le dispositive pédagogique de la classe inversée « flipped classroom » a suscité l'intérêt de plusieurs chercheurs dans le domaine de l'enseignement/apprentissage à des niveaux différents de la scolarité, notamment au niveau universitaire. Ce dispositif pédagogique s'oppose à la méthode traditionnelle prédominante dans l'enseignement universitaire qui est basée sur un cours magistral où l'étudiant est la plupart du temps passif et son rôle se limite à la prise de notes de cours. Les séances de travaux dirigées où l'étudiants acquiert un rôle plus actif ont pour objectif la correction des exercices et problèmes relatifs au cours magistral et qui ont été proposés comme un devoir (homework). La classe inversée inverse ce dispositif dans un objectif d'accroitre l'interaction entre l'enseignant et l'apprenant et d'attribuer à l'étudiant un rôle plus actif dans ses apprentissages sous la direction de l'enseignant. Elle vise ainsi permettre à l'étudiant d'être plus autonome et d'apprendre à son propre rythme. La pratique de la classe inversée comprend deux parties ; une partie hors classe (the out-of-class part) dans laquelle le cours est proposé aux apprenants comme devoir par le biais de documents ou de vidéos sélectionnées ou élaborées par l'enseignant. La deuxième partie est la partie en classe qui est consacrée aux activités de résolution de problèmes. Les travaux de recherches sur la classe inversée, indiquent une grande variété dans l'implémentation de ce dispositif. Bishop et Verleger [5] indiquent deux principes de ce dispositif : (1) introduire le contenu mathématique visé à travers des capsules vidéos à visualiser individuellement en utilisant un ordinateur ou un autre appareil. (2) des activités collaboratives en classe pour la résolution des exercices proposés.

Plusieurs résultats positifs ont été reportés dans des travaux de recherches en lien avec l'augmentation dans la motivation des apprenants lors de la mise en place du dispositif de 
classe inversée [6-10]. L'aspect positif des résultats est essentiellement lié à l'évolution de l'engagement social des étudiants dans leur apprentissage, ainsi qu'à la flexibilité fournie à travers les vidéos proposées. D'autres travaux $[11,12,13]$ prennent une position plutôt critique par rapport à cette nouvelle approche. Les résultats de l'étude comparative réalisée par Strayer [11] entre deux groupes d'étudiants, l'un ayant suivi un enseignement classique et l'autre un enseignement mettant en œuvre la pratique de la classe inversée, font apparaitre que contrairement aux étudiants ayant suivi un enseignement classique, ceux ayant expérimenté la classe inversée se sont sentis « perdus » et désengagés. Dans son travail de thèse, Ramaglia [12] a comparé les résultats de deux groupes d'étudiants dans l'examen commun de fin de semestre, l'un ayant suivi un enseignement classique et l'autre un enseignement basé sur la stratégie de la classe inversée. Elle a également étudié la différence entre les perceptions des étudiants et des enseignants sur les résultats en mathématiques pour les deux groupes. Ses analyses ont permis de pointer et de décrire des incidents associés à l'apprentissage actif en milieu scolaire. Bridoux [13] a expérimenté le dispositif de classe inversée au niveau de la première année universitaire pour l'introduction de la notion de suite. Les résultats de son expérience font apparaitre les difficultés des étudiants à tirer profit des capsules vidéos proposées par l'enseignant en absence d'un contrat didactique explicite. Bridoux [13] considère qu'il serait nécessaire de passer du temps à montrer aux étudiants comment travailler à la maison avec ce matériel, en les incitant par exemple à prendre des notes de manière à rester actifs pendant le visionnage de la capsule ou en rappelant que le fait d'apprendre le cours avec une capsule ne modifie pas les exigences de l'enseignant dans les productions des étudiants [13].

Plusieurs cadres théoriques ont été utilisés dans les travaux de recherches ayant abordé la problématique de l'utilisation du dispositif de la classe inversée. Les cadres les plus utilisés sont essentiellement, la TAD, la théorie de l'activité [13] et Realistic Mathematics Education (RME) [9, 10]. Dans notre travail nous nous plaçons dans le cadre de l'approche commognitive qui conceptualise les mathématiques comme un discours et leur apprentissage comme une appropriation du discours à travers l'individualisation de l'ensemble des routines introduites pour l'apprentissage d'un objet mathématique. Ce choix revient aux outils théoriques et analytiques fournies par ce cadre théorique permettant d'étudier l'impact de l'utilisation du dispositif de la classe inversée sur l'apprentissage des mathématiques. L'objet du paragraphe suivant est de donner une présentation sommaire du cadre théorique utilisé et de justifier son apport à notre étude.

\section{Cadre théorique}

La théorie commognitive [14] est une approche discursive qui conceptualise l'apprentissage des mathématiques dans sa dimension socioculturelle. Cette approche définit les mathématiques comme une activité de communication [15] et l'apprentissage des mathématiques comme un développement du discours. Selon cette approche, le discours mathématique sur les séries de Fourier est défini comme un type de communication utilisé pour élaborer des récits à propos des séries de Fourier. Ainsi, ce discours émerge lorsque les étudiants sont engagés dans une communication avec les autres ou avec eux-mêmes à propos de ces séries. Le discours mathématique sur les séries de Fourier, comme tout autre discours mathématique, est caractérisé par quatre propriétés : le vocabulaire utilisé, les médiateurs visuels, les récits approuvés et les routines.

Le vocabulaire utilisé : inclut le vocabulaire mathématique relatif aux séries de Fourier (développement en série de Fourier, convergence de la série, coefficients de Fourier, pulsation...)

Les médiateurs visuels : Sfard [14] décrit les médiateurs visuels comme des objets visibles à travers lesquels se concrétise la communication. Tabach et Nachlieli, [16] considèrent que 
ces médiateurs visuels, en tant qu'outil de représentation, peuvent influencer la pensée et les actions des interlocuteurs. Dans le discours relatif aux séries de Fourier, nous distinguons trois catégories de médiateurs visuels :

Les médiateurs visuels symboliques : qui comportent à titre d'exemples, la pulsation $\omega=\frac{2 \pi}{T}$, les expressions des coefficients de Fourier $a_{0}=\frac{1}{T} \int_{a}^{a+T} f(t) d t, a_{n}=$ $\frac{2}{T} \int_{a}^{a+T} f(t) \cos (n \omega t) d t, b_{n}=\frac{2}{T} \int_{a}^{a+T} f(t) \sin (n \omega t) d t$ ainsi que le développement en série de Fourier d'une fonction périodique $a_{0}+\sum_{n=1}^{+\infty} a_{n} \cos (\mathrm{n} \omega x)+b_{n} \sin (n \omega x)$

Les médiateurs visuels graphiques : comme les représentations graphiques des fonctions périodiques étudiées ainsi que les sommes partielles de la série de Fourier.

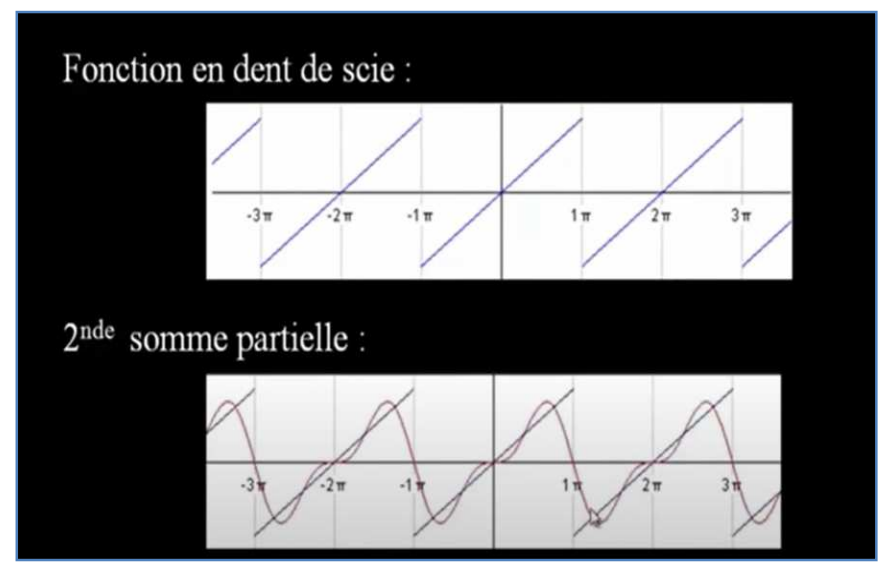

Fig. 1. Un exemple des médiateurs visuels graphiques relatifs à la fonction en dent de scie et une de ses approximations.

Les médiateurs visuels dynamiques : ce sont les représentations graphiques ayant un aspect dynamique. Parmi les médiateurs visuels proposés aux étudiants, les différentes approximations d'un signal par les différentes sommes partielles ${ }^{\dagger}$.

Les récits approuvés : qui comprennent les textes écrits ou oraux décrivant les objets et les processus ainsi que les relations entre eux et qui sont soumis à la validation, à la modification ou au rejet selon des règles définies par la communauté. Nous citons comme exemples les définitions, théorèmes et preuves (théorème de Dirichlet, égalité de Parseval, ...)

Les routines comprennent les pratiques régulièrement utilisées et bien définies par la communauté (telles que la définition, la conjecture, la preuve, l'estimation, la généralisation et l'abstraction). Dans l'objectif de rendre la notion de routine plus opérationnelle Lavie, Steiner et Sfard, [17] et Lavie et Sfard, [18] décrivent une routine comme une paire tâcheprocédure. La tâche indique ce qui doit être réalisé en exécutant la routine (ex. produire un récit approuvable sur la convergence d'une série de Fourier.) La procédure est une prescription sur la façon dont cela pourrait être fait. Dans cette perspective, ces auteures conceptualisent l'apprentissage comme un processus de routinisation des actions des apprenants. Cette façon d'approcher l'enseignement des mathématiques est basée sur l'hypothèse que lors des premières rencontres avec un nouveau discours relatif à une nouvelle notion mathématique, les apprenants ne peuvent participer à ce discours que de manière ritualisée. Par la suite et dans l'évolution de leurs apprentissages, leurs routines devraient

\footnotetext{
${ }^{\dagger}$ https://upload.wikimedia.org/wikipedia/commons/d/d4/Synthesis_sawtooth.gif
} 
subir une dé-ritualisation progressive jusqu'à ce qu'elles se transforment en véritables explorations.

Ces quatre caractéristiques du discours ne sont pas indépendantes. En fait, le discours relatif aux séries de Fourier est lié à l'élaboration d'un ensemble de récits à propos de cet objet mathématique. L'élaboration de ces récits nécessite l'utilisation du vocabulaire associé ainsi que l'ensemble de médiateurs visuels permettant d'éclairer et d'appuyer ces récits. Les routines constituent les règles qui régissent leur élaboration et leur validation.

Pour étudier l'impact de l'utilisation du dispositif de classe inversée sur l'apprentissage des séries de Fourier de point de vue de l'approche commognitive et en termes de routines, nous commençons par expliciter les routines proposées dans les capsules vidéo et puis nous chercherons à étudier leur individualisation par les étudiants. Le travail est guidé par les questions de recherches suivantes : Quelles sont les caractéristiques des routines relatives aux séries de Fourier introduites par les capsules vidéos ? Comment permettent-elles aux étudiants de donner un sens à ces séries? Comment ces caractéristiques influencent-elles l'apprentissage des séries de Fourier par des étudiants de première année?

\section{Description du déroulement de l'expérience}

\subsection{Contexte institutionnel}

L'expérience a été réalisée au deuxième semestre de l'année universitaire 2018/2019 à la faculté des sciences de Bizerte en Tunisie avec la participation de 68 étudiants de première année en sciences de l'informatiques, habitués à un enseignement classique qui se présente sous forme de séances de cours magistraux suivies par des séances de travaux dirigées destinées à la correction des séries d'exercices proposés. Nous signalons que ce travail a constitué la première expérience de la classe inversée à la fois pour l'enseignant-chercheur que nous sommes et pour les étudiants.

\subsection{Dispositif expérimental}

Le cours est réparti en un ensemble de 10 capsules vidéos provenant du site mathenvidéo La durée des vidéos varie entre 54 secondes et $4 \mathrm{mn} 40$ secondes. Les capsules sont organisées de façon que chacune correspond à un seul objectif et par conséquence, introduit une seule routine. Ces vidéos peuvent être classées en deux catégories. La première catégorie comporte les vidéos relatives à la présentation du savoir. Les quatre premières vidéos sont relatives cette catégorie. Dans ces vidéos, il y a une introduction du vocabulaire relatif aux séries de Fourier, les médiateurs visuels graphiques, symboliques et dynamiques ainsi que l'interprétation graphique du développement en série de Fourier d'une fonction périodique. La deuxième catégorie comporte les vidéos relatives aux savoir-faire à acquérir par les étudiants. Ces vidéos proposent les routines visées par l'enseignement des séries de Fourier. Cette catégorie comporte les 6 vidéos suivantes qui présentent un exemple d'application avec une fonction constante par morceaux. Ces vidéos explicitent les différentes étapes et stratégies à adopter pour le développement d'une fonction périodique en une série de Fourier.

\footnotetext{
${ }^{*}$ https://www.mathenvideo.fr/category/bts-2nde-annee/serie-de-fourier/developper-enserie-de-fourier/
} 


\subsection{Déroulement de l'expérience}

L'expérimentation a démarré avec une séance présentielle pendant laquelle nous avons présenté le projet et les principes de base de la classe inversée. Cette séance a été suivie par une mise en ligne des quatre premières vidéos sur la plateforme Moodle. Aucune consigne spécifique n'a été donnée à part le fait que ces vidéos remplacent le cours magistral.

La première séance présentielle a commencé par un premier questionnaire d'investigation sur la prise de contact avec les vidéos (le nombre de fois qu'ils ont visualisé les vidéos et s'ils ont pris des notes ou non) ainsi que sur le contenu scientifique de ces vidéos. Par la suite, la séance s'est transformée sous forme d'une discussion et d'interaction avec les étudiants à propos du contenu des vidéos déposées. La discussion a consisté à répondre aux questions des étudiants à propos du contenu. L'étape suivante a consisté à déposer sur la plateforme Moodle les 6 vidéos suivantes du cours. Les vidéos ont été suivies par un questionnaire en ligne. Au niveau de cette étape, et à la suite de l'analyse des données recueillies à travers le premier questionnaire, nous avons exigé aux étudiants la prise de notes de cours qui seront à remettre la séance suivante. Une semaine après, pendant la séance en classe et lors de la résolution des problèmes, nous avons constaté une hétérogénéité importante chez les étudiants. Cela a obligé l'enseignante que nous sommes, à la transformer en une séance de synthèse où le contenu scientifique des vidéos a été repris et synthétisé. Les deux séances de travaux dirigées suivantes en classe, étaient consacrées à des activités collectives de résolution de problèmes. L'expérience a été clôturée par un questionnaire final qui vise l'exploration de l'individualisation par les étudiants des routines proposées dans les capsules vidéos et la possibilité de leurs mobilisations lors de la résolution des tâches proposées dans le questionnaire. Le paragraphe suivant comporte les résultats de l'analyse pédagogique et l'analyse didactique de l'expérimentation. L'analyse pédagogique vise l'étude du fonctionnement du dispositif de la classe inversée pour un éventuel ajustement. L'analyse didactique vise l'étude de l'individualisation par les étudiants des routines introduites dans les capsules vidéos qui présentent le cours sur les séries de Fourier.

\section{Analyse pédagogique : le fonctionnement du dispositif de la classe inversée}

\section{Questionnaire 1: une première investigation du fonctionnement du dispositif de la classe inversée}

Le premier questionnaire a pour objectif d'explorer le fonctionnement du dispositif de la classe inversée. Le questionnaire comporte deux parties. La première partie est relative à la prise en contact des étudiants avec les vidéos à travers le nombre de visualisations de ces vidéos et la prise de notes de cours. La deuxième partie explore l'appropriation du contenu des quatre premières vidéos par les étudiants. L'analyse des données recueillies par ce questionnaire a permis de nous informer sur les besoins potentiels d'ajustement lors de la deuxième phase de l'expérimentation.

Partie 1 : Prise en contact avec les vidéos

Le tableau suivant synthétise les réponses des étudiants à propos des nombres de fois où ils ont visualisé les vidéos et s'ils ont pris ou non des notes de synthèse :

1. Combien de fois avez-vous visualisé les vidéos du cours?

2. Y-a-t-il une ou des vidéos que vous avez regardées plus de fois que les autres ? Lesquelles et pourquoi ?

3. Avez-vous pris des notes en regardant les vidéos?

Si NON expliquez pourquoi ?

Si OUI Qu'avez-vous noté ? 
Tableau 1. Résultats de la partie 1 du questionnaire.

\begin{tabular}{|c|c|}
\hline $\begin{array}{l}\text { Nombre de fois } \\
\text { les vidéos ont } \\
\text { été visualisées }\end{array}$ & $\begin{array}{l}\text { Nombre } \\
\text { d'étudiants }\end{array}$ \\
\hline 0 fois & $\begin{array}{c}14 \\
(22.87 \%)\end{array}$ \\
\hline 1 fois & $\begin{array}{c}15 \\
(23.43 \%)\end{array}$ \\
\hline $2-3$ fois & $\begin{array}{c}19 \\
(29.68 \%)\end{array}$ \\
\hline Plus que 3 fois & $\begin{array}{c}16 \\
(25 \%)\end{array}$ \\
\hline
\end{tabular}

Les résultats font apparaitre des comportements variables chez les étudiants en lien avec la manipulation et l'utilisation des capsules vidéos. Les données montrent qu'un nombre important des étudiants (14 parmi les 64 soit 22.87\%) n'ont pas regardé les vidéos pour l'une des raisons suivantes : Soit, ils étaient absents lors de la séance d'information, soit, ils avaient un problème de connexion Internet. 15 étudiants parmi les 64 participants ont regardé les vidéos une seule fois. Ce qui est à notre avis insuffisant pour s'approprier le contenu proposé. Nous considérons que ces résultats traduisent un problème au niveau de l'autonomie des étudiants dans ce nouveau dispositif ce qui peut avoir comme conséquence que les étudiants ne profitent pas du contenu proposé dans les vidéos.

Dans les réponses au questionnaire, seuls 15 étudiants ont déclaré qu'ils ont pris des notes à partir des vidéos. Ces notes concernent essentiellement l'utilité des séries de Fourier et la formule générale du développement en série de Fourier. Nous remarquons ainsi que les étudiants qui étaient habitués à prendre systématiquement des notes lors des séances de cours, ne voient pas l'utilité de prendre des notes de cours à partir des vidéos. Nous considérons que les étudiants trouvent des difficultés au niveau de ce changement important du degré d'autonomie dans ce nouveau dispositif. Cette première partie du questionnaire nous a permis d'identifier l'importance de guider les étudiants dans la partie hors-classe pour imposer une lecture active des vidéos. Pour cela nous avons choisi d'imposer aux étudiants l'élaboration de notes de synthèse du contenu proposé dans les vidéos et nous avons donné un deuxième questionnaire en ligne.

Partie 2: Appropriation du vocabulaire utilisé dans les premières vidéos

L'objectif de la deuxième partie du questionnaire proposé vise l'exploration de l'appropriation du vocabulaire utilisé dans les vidéos. Cette partie se présente sous forme d'un QCM composé de trois questions :

1/ A quoi servent les séries de Fourier?

- Etude de phénomènes vibratoires.

- Calculer des sommes infinies.

- Etudier les fonctions cosinus et sinus.

- Faire des approximations des fonctions périodiques.

2/ On considère une fonction périodique de période $\mathrm{T}$ et sa décomposition en série de Fourier :

$$
a_{0}+\sum_{n=1}^{+\infty} a_{n} \cos (n w t)+b_{n} \sin (n w t)
$$

$a_{0} ; a_{n}$ et $b_{n}$ sont appelés :

- Les facteurs de Fourier 
- Les pulsations de Fourier

- Les coefficients de Fourier

3/ Répondre par vrai ou faux en justifiant la réponse

- $\quad$ Si la fonction $f$ est paire alors $a_{n}=0$

- Si la fonction $f$ est paire alors $b_{n}=0$

- Si la fonction $f$ est impaire alors $a_{n}=0$

Les résultats des réponses à cette partie du questionnaire sont synthétisés dans le tableau suivant :

Tableau 2. Résultats de la partie 2 du questionnaire.

\begin{tabular}{|l|c|c|c|}
\hline $\begin{array}{c}\text { Numéro de la } \\
\text { question }\end{array}$ & $\begin{array}{c}\text { Réponse } \\
\text { correcte }\end{array}$ & $\begin{array}{c}\text { Réponse } \\
\text { erronée }\end{array}$ & Pas de réponse \\
\hline Q1 & 29 & 14 & 21 \\
& $45,31 \%$ & $21,87 \%$ & $32,81 \%$ \\
\hline Q2 & 35 & 10 & 19 \\
& $54,69 \%$ & $15,62 \%$ & $29,69 \%$ \\
\hline Q3 & 16 & 28 & 20 \\
& $25 \%$ & $43,75 \%$ & $31,25 \%$ \\
\hline
\end{tabular}

Les résultats font apparaitre une importante hétérogénéité chez les étudiants. Cette hétérogénéité se traduit par l'identification de trois groupes d'étudiants : des étudiants n'ayant aucune idée sur le contenu puisqu'ils n'ont pas regardé les vidéos, des étudiants incapables d'identifier le vocabulaire utilisé dans les vidéos puisque le nombre de fois où ils ont visualisé le contenu est insuffisant pour s'approprier ce vocabulaire et des étudiants ayant répondu correctement au questionnaire et nous supposons qu'ils ont fait des lectures actives des vidéos.

Ce premier questionnaire avait pour objectif d'évaluer le fonctionnement du nouveau dispositif de la classe inversée chez les étudiants pour des éventuels ajustements. L'analyse de la première prise en contact avec ce dispositif nous a permis d'identifier des résistances de la part des étudiants et des difficultés à s'adapter avec l'importante variation au niveau du degré d'autonomie dans l'appropriation du contenu mathématique proposé. Cela nous a amené à conclure la nécessité de guider les étudiants dans la phase out-of-class à travers l'exigence de prise de notes à partir des capsules vidéos et la proposition de QCM afin de leur imposer une lecture active du contenu proposé.

L'hétérogénéité au niveau de l'appropriation du contenu lors de la première séance présentielle, nous a permis de conclure la nécessité d'un enseignement hybride du contenu. Pour cela une séance de synthèse en présentiel a été programmée. Cette séance a pour objectif de reprendre le vocabulaire relatif aux séries de Fourier et des médiateurs visuel symboliques ainsi que les théorèmes associés.

\section{Analyse didactique : individualisation des routines par les étudiants}

\subsection{Analyse des routines visées par les vidéos}

Les vidéos proposées visent l'installation d'un certain nombre de routines relatives aux séries de Fourier. La notion de « routines visées » est définie comme étant «l'ensemble des routines proposées dans un objectif d'enseignement d'une notion mathématique spécifique. [...] Ce sont les routines telles qu'elles sont susceptibles d'être interprétées et réalisées par 
un expert en utilisant les connaissances relatives au niveau scolaire en question » [19]. L'analyse des vidéos nous a permis de classer les routines visées selon trois catégories : les routines de traitement calculatoire, les routines de traitement graphique et les routines de justification et de preuve. Pour chacune des routines une procédure typique est proposée dans la vidéo que les étudiants sont supposés utiliser lors de la résolution des activités en classe.

Tableau 3. Les trois catégories des routines visées dans les capsules vidéos.

\begin{tabular}{|c|c|c|}
\hline $\begin{array}{l}\text { Les routines de traitement } \\
\text { calculatoire }\end{array}$ & $\begin{array}{l}\text { Les routines de traitement } \\
\text { graphique }\end{array}$ & $\begin{array}{ccc}\text { Les routines } & \text { de } \\
\text { justification et preuve }\end{array}$ \\
\hline $\begin{array}{l}\text { Calculer la pulsation } \omega=\frac{2 \pi}{T} \\
\text { Calculer les coefficients de } \\
\text { Fourier relatifs à un signal } \\
\text { périodique. } \\
\text { DDévelopper une fonction } \\
\text { périodique en série de Fourier. } \\
\text { Détermination de la valeur } \\
\text { efficace d'un signal. } \\
\text { Calcul de certaines valeurs de } \\
\text { la somme partielle. }\end{array}$ & $\begin{array}{l}\text { DConstruire la représentation } \\
\text { graphique d'un signal. } \\
\text { Interprétation graphique de } \\
\text { l'approximation d'un signal } \\
\text { par une série de Fourier. } \\
\text { DDétermination graphique } \\
\text { de la période. } \\
\text { Interprétation graphique de } \\
\text { la valeur moyenne de la série } \\
\text { de Fourier. }\end{array}$ & $\begin{array}{l}\square \text { Étudier la convergence } \\
\text { simple de la série de Fourier } \\
\text { en utilisant le théorème de } \\
\text { Dirichlet. } \\
\square \text { Étudier la convergence } \\
\text { uniforme de la série de } \\
\text { Fourier. } \\
\square \text { Établir des formules de } \\
\text { sommes de séries en } \\
\text { appliquant la convergence en } \\
\text { des valeurs particulières. }\end{array}$ \\
\hline
\end{tabular}

1/ Routines de traitement graphique : ce sont les routines relatives à l'utilisation des médiateurs visuels graphiques. Nous distinguons :

- Les routines de construction de la représentation graphique de la fonction périodique donnée qui représente le signal. Ces routines constituent une généralisation de routines déjà rencontrées par les étudiants lors de l'étude des fonctions trigonométriques au niveau du lycée et dans le module d'Analyse 1 durant le premier semestre. Cependant, la nouveauté réside essentiellement au niveau de la rencontre de nouvelles fonctions périodiques (la fonction dents de scie, la fonction créneau, ...) ce qui nécessite l'application des procédures déjà connues à ces nouvelles fonctions. Cela constitue une extension au niveau du domaine d'application de ces routines de manipulation graphique. Notons que la propriété de périodicité n'a pas était investie dans l'enseignement secondaire puisque lorsque les fonctions étudiées sont périodiques, le travail est réduit à un intervalle d'une période, imposé dans l'énoncé des exercices proposées. De plus, dans le module d'Analyse 1, les médiateurs visuels graphiques sont utilisés uniquement dans la partie cours pour illustrer l'introduction des fonctions trigonométriques réciproques [19]. La vidéo 5 explique les procédures à utiliser pour accomplir la tâche de représentation graphique de la fonction étudiée qui exploitent les propriétés de parité et de périodicité.

- Les routines d'interprétation graphique de l'approximation d'un signal par une série de Fourier. Ces routines sont introduites au niveau de la vidéo 3 intitulée «Interprétation graphique du développement en série de Fourier d'une fonction périodique donnée ». Cette vidéo donne les représentations graphiques de certaines sommes partielles de la série de Fourier de la fonction représentée. La série de Fourier est ainsi introduite en tant qu'une approximation d'une fonction périodique donnée par une somme de sinus et de cosinus affectés de coefficients. Cette interprétation graphique associée aux séries de Fourier qui est une notion ayant un aspect formel important avec un niveau de symbolisme assez poussé, permet de lui donner une signification, de justifier son introduction (sa raison d'être) ainsi que permettre aux étudiants de lui associer une image mentale. L'utilisation du dispositif de la classe inversée permet à travers l'utilisation de ces vidéos d'introduire ces routines graphiques difficiles à utiliser et à visualiser dans le cadre d'un enseignement magistral (classique) ce qui constitue un apport important à l'enseignement de la notion de séries de 
Fourier qui permet de motiver les étudiants et de leur permettre d'avoir une interprétation intuitive de cette notion.

2/ Routines de traitement calculatoire : Ce sont les routines relatives au calcul de la pulsation d'un signal donné, la détermination des coefficients de Fourier d'une fonction périodique donnée ainsi que le développement d'une fonction périodique en série de Fourier. Les vidéos proposées explicitent les procédures de calcul de ces coefficients utilisant les propriétés des intégrales ainsi que les propriétés de parité et de périodicité de la fonction étudiée. Cette catégorie englobe également la détermination de la valeur efficace d'un signal et son interprétation afin de mettre en évidence l'utilité des séries de Fourier dans le domaine de la physique, du traitement du signal et du traitement de l'image et cela essentiellement au niveau de certaines activités proposées aux étudiants.

\section{3/ Routines de justification et de preuve :}

Ces routines sont très utilisées dans les activités et les exercices proposés aux étudiants lors de la phase de travail en classe. Parmi ces routines nous avons identifié deux types de routines visées dans les vidéos. Le premier type concerne les routines relatives à l'étude de la convergence simple ou uniforme de la série de Fourier en utilisant le théorème de Dirichlet. Le second type concerne les routines de détermination de la somme d'une série numérique convergente à travers l'application du théorème de Dirichlet ou l'égalité de Parseval pour des valeurs particulières.

Pour l'exploration de l'individualisation de ces routines par les étudiants à travers l'analyse de leurs réponses au questionnaire final proposé à la fin de l'expérimentation, nous focalisons notre attention sur l'individualisation des routines graphiques afin d'identifier l'impact de l'introduction de routines de traitement graphique à travers le dispositif de la classe inversée sur l'apprentissage de la notion de séries de Fourier. Nos analyses concernent ainsi les réponses des étudiants au troisième exercice du questionnaire final.

\subsection{Résultats du questionnaire final : étude de l'individualisation des routines de traitement graphique}

L'objet de cet exercice est d'étudier l'individualisation des routines graphiques par les étudiants. Pour cela nous avons cherché à identifier si les étudiants sont capables de reconnaitre les caractéristiques des séries de Fourier des fonctions périodiques données à partir de l'interprétation de leurs représentations graphiques. Cela permettra de repérer s'ils sont capables de donner des conclusions sur les coefficients des séries de Fourier et sur la convergence de ces séries en les reliant à des données graphiques comme la parité de la fonction, sa périodicité et sa continuité. Ainsi, dans les questions Q1, Q2, Q3 et Q4, l'étudiant est amené à identifier à partir de la représentation graphique si la fonction donnée est paire, impaire, ou impaire symétrique pour pouvoir conclure lequel des coefficients de Fourier est nul. Au niveau des deux questions Q5 et Q6, il s'agit d'identifier la continuité de la fonction donnée à partir de sa représentation graphique et de relier cela au théorème de Dirichlet pour la convergence simple ou uniforme des séries de Fourier. Pour la justification, l'étudiant doit s'appuyer sur les énoncés déjà rencontrées dans les capsules vidéos et utilisés lors des séances de résolution de problèmes. 


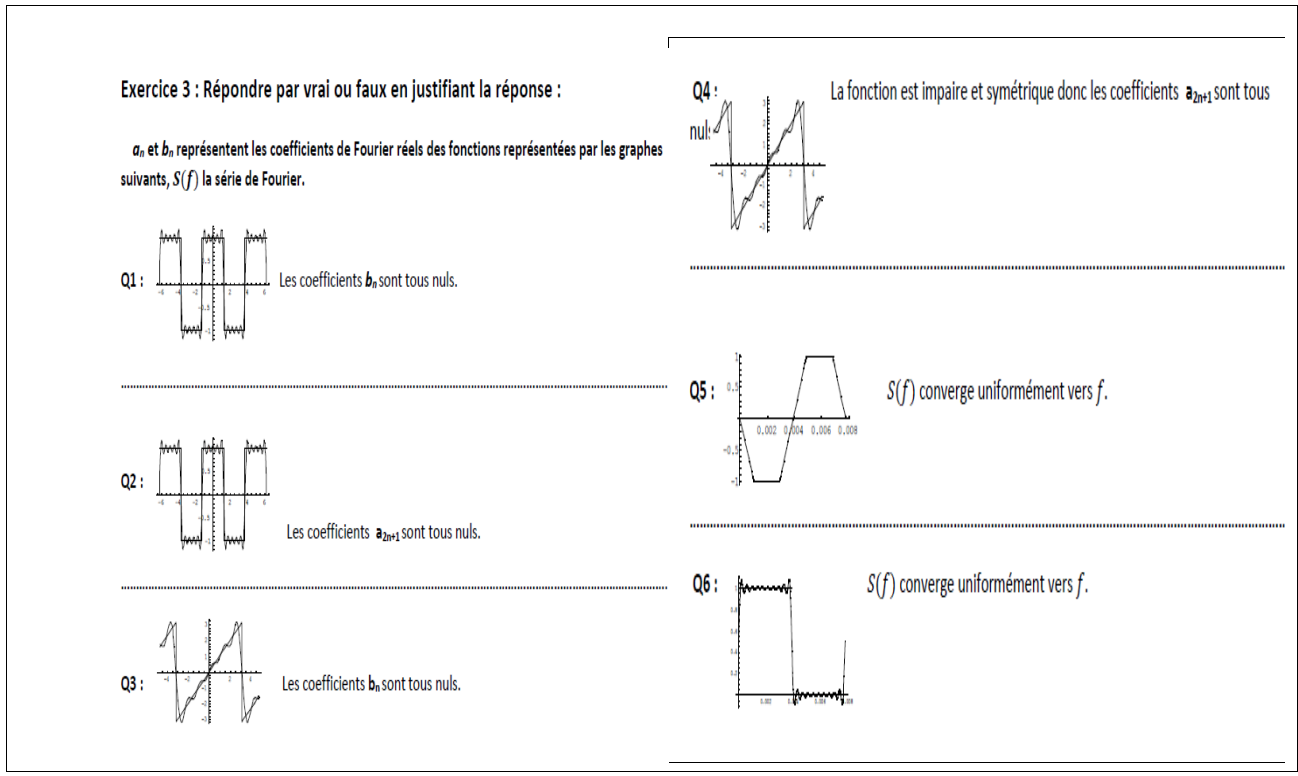

Fig. 2. Enoncé de l'exercice 3 du questionnaire final.

Notons que seuls 60 étudiants parmi les 64 ont passé le questionnaire final (les quatre autres étudiants étaient absents). Les résultats de l'analyse des réponses sont résumés dans le tableau 4.

Tableau 4. Résultats de l'analyse des réponses à l'exercice 3 du questionnaire final.

\begin{tabular}{|l|c|c|c|}
\hline & $\begin{array}{c}\text { Réponse } \\
\text { correcte }\end{array}$ & Réponse fausse & Pas de réponse \\
\hline Question 1 & $\mathbf{4 3}$ & $\mathbf{1 4}$ & $\mathbf{3}$ \\
& $\mathbf{7 1 , 6 6 \%}$ & $\mathbf{2 3 , 3 3 \%}$ & $\mathbf{5 \%}$ \\
\hline Question 2 & $\mathbf{4 1}$ & $\mathbf{1 5}$ & $\mathbf{4}$ \\
& $\mathbf{6 8 , 3 3 \%}$ & $\mathbf{2 5 \%}$ & $\mathbf{6 , 6 6 \%}$ \\
\hline Question 3 & $\mathbf{4 2}$ & $\mathbf{1 8}$ & $\mathbf{0}$ \\
& $\mathbf{7 0 \%}$ & $\mathbf{3 0 \%}$ & $\mathbf{0 \%}$ \\
\hline Question 4 & $\mathbf{4 1}$ & $\mathbf{1 4}$ & $\mathbf{5}$ \\
& $\mathbf{6 8 , 3 3 \%}$ & $\mathbf{2 3 , 3 3 \%}$ & $\mathbf{8 , 3 3 \%}$ \\
\hline Question 5 & $\mathbf{4 2}$ & $\mathbf{1 2}$ & $\mathbf{6}$ \\
& $\mathbf{7 0 \%}$ & $\mathbf{2 0 \%}$ & $\mathbf{1 0 \%}$ \\
\hline Question 6 & $\mathbf{2 2}$ & $\mathbf{3 0}$ & $\mathbf{8}$ \\
& $\mathbf{3 6 , 6 6 \%}$ & $\mathbf{5 0 \%}$ & $\mathbf{1 3 , 3 3 \%}$ \\
\hline
\end{tabular}

L'analyse des réponses des étudiants a permis de noter l'importance du taux de réussite des questions de l'exercice 3 du questionnaire. Ces questions font appel à une interprétation graphique des fonctions périodiques et du développement en série de Fourier de ces fonctions. En fait, le taux de réussite des questions 1, 2, 3 et 4 varie entre $68,33 \%$ et $71,66 \%$. Ces résultats permettent de noter que les étudiants sont capables d'identifier les propriétés des coefficients de Fourier à partir de l'interprétation des représentations graphiques et donc sont capables de relier les données graphiques aux coefficients de Fourier (notions définies de façon formelle). L'importance du taux de réussite au niveau de la question 5 permet de conclure que les étudiants sont capables de reconnaitre la convergence simple ou uniforme de la série de Fourier à partir d'une interprétation graphique et cela en identifiant la continuité 
de la fonction et en appliquant le théorème de Dirichlet. Le taux de réussite faible au niveau de la $6^{\text {ème }}$ question par rapport aux questions précédentes revient essentiellement au fait que les étudiants étaient incapables de repérer les points de discontinuité à cause de la présence de la représentation graphique de l'approximation par une somme partielle. Cela nous amène à considérer que les routines de traitement graphique sont mobilisées par la plupart des étudiants et par conséquence que ces étudiants ont individualisé ce type de routine. Cela constitue un exemple d'un effet positif du dispositif de la classe inversée. Cependant nous avons également remarqué que certains étudiants trouvent des difficultés à donner une justification à leurs réponses et se limitent à des justifications intuitives ou graphiques. Nous pouvons ainsi avancer une première conclusion à propos des routines de preuve et de justification: Les routines de justification basées sur une justification graphique sont bien réussies par les étudiants. Cependant, les routines de preuve restent une source de difficultés importante.

\section{Conclusions générales}

Dans ce travail nous avons analysé une séquence d'enseignement basée sur l'utilisation du dispositif de la classe inversée pour introduire la notion de série de Fourier dans un cours de mathématiques destiné à des étudiants de première année en science de l'informatique. Les analyses ont porté sur deux niveaux : le premier niveau s'intéresse au fonctionnement du dispositif de la classe inversée et le second niveau constitue une analyse didactique de l'impact de ce dispositif sur l'apprentissage des étudiants. Dans ce papier, nous avons focalisé notre attention sur l'individualisation des routines de traitement graphique introduites dans les capsules vidéos proposées aux étudiants dans la phase « hors classe ».

L'étude du fonctionnement du dispositif de la classe inversée, nous a permis d'affirmer que l'autonomie des étudiants dans la phase « hors-classe » n'est pas garantie et nécessite d'être orientée par l'enseignant. En fait, nous considérons qu'il est nécessaire d'expliciter et de mettre en place un nouveau contrat didactique pour une utilisation active des vidéos « hors-classe » et cela en imposant la prise de notes et en proposant des questionnaires qui imposent aux étudiants le retour vers le contenu proposé dans les vidéos afin de répondre aux questions. L'analyse du fonctionnement du dispositif de la classe inversée nous a permis de souligner l'importance de mettre en place des séances de synthèse dans la phase « en-classe » afin de mettre le point sur le vocabulaire spécifique introduit, les définitions et théorèmes. Cela permet ainsi de faire le lien avec l'enseignement classique auquel les étudiants sont attachés et qui engendre chez certains étudiants une certaine résistance au nouveau dispositif. Cette résistance est apparue à travers la non-lecture des capsules vidéos ou leur visualisation une seule fois, ce qui ne leur permet pas d'identifier les routines visées dans le contenu proposé. Cela nous permet de conclure l'importance du rôle de l'enseignant pour guider le processus d'apprentissage dans les deux phases « en-classe » et « hors-class »

Le deuxième niveau d'analyse est de type didactique et vise l'étude de l'individualisation des routines proposées dans les vidéos. Dans ce papier nous nous sommes limitées à l'étude des routines de traitement graphique. Nous avons ainsi dégagé que le dispositif de la classe inversée a permis aux étudiants de faire le lien entre les réalisations symboliques et graphiques des séries de Fourier et de donner un sens à cette notion.

L'enseignement classique permet aux étudiants de s'approprier les routines de calcul et de convergence des séries de Fourier. Cependant, l'utilisation des vidéos dans le dispositif de la classe inversée a permis aux étudiants de lui donner un sens.

Les démonstrations dans les problèmes de convergence des séries de Fourier et de calcul des valeurs des séries restent source de plusieurs difficultés chez les étudiants, bien que les séances de travail collectif en classe étaient basées sur ce type d'exercices. Nous considérons 
que plus de travail est nécessaire pour la conception et l'analyse des phases de résolution des activités en classe.

\section{Références}

1. M. J. C. S. Reis, S. Soares, S. Cardeal, R. Morais, E. Peres, P. J. S. G. Ferreira, Teaching of Fourier series expansions in undergraduate education, in 2013 IEEE Global Engineering Education Conference (EDUCON), Berlin, p. 431-435, (2013) doi: 10.1109/EduCon.2013.6530141.

2. P. Avitabile, J. Hodgkins, T. Van Zandt, A. Butland, D. Nicgorski, Innovative teaching of Fourier series using LabView, (2006).

3. L. Alcock, A. Simpson, Convergence of sequences and series: Interactions between visual reasoning and the learner's beliefs about their own role, ESM, 57, $\mathrm{n}^{\mathrm{o}}$ 1, p. 1-32, (2004)

4. A. S. González-Martín, E. Nardi, I. Biza, conceptually driven and visually rich tasks in texts and teaching practice: the case of infinite series, IJMEST, vol. 42, $\mathrm{n}^{0} 5, \mathrm{p} .565-$ 589, (2011)

5. J. L. Bishop, M. A. Verleger, The flipped classroom: A survey of the research, in ASEE national conference proceedings, Atlanta, GA, 30, p. 1-18. (2013)

6. V. N. Franqueira, P. Tunnicliffe, To flip or not to flip: A critical interpretive synthesis of flipped teaching, in Smart Education and Smart e-Learning, Springer, p. 57-67. (2015)

7. S. Kadry, A. El Hami, Flipped classroom model in calculus II, Education, 4, no 4, p. 103-107, (2014)

8. S. Roshan, The flipped classroom: touch enabled, academically proven, in The Impact of Pen and Touch Technology on Education, Springer, p. 215-222, (2015)

9. M. Voigt, H. Fredriksen, C. Rasmussen, Leveraging the design heuristics of realistic mathematics education and culturally responsive pedagogy to create a richer flipped classroom calculus curriculum, ZDM, p. 1-12, (2020)

10. H. Fredriksen, Exploring Realistic Mathematics Education in a Flipped Classroom Context at the Tertiary Level, IJSME, p. 1-20, (2020)

11. J. F. Strayer, How learning in an inverted classroom influences cooperation, innovation and task orientation, $L E R, 15, \mathrm{n}^{\circ} 2$, p. 171-193, (2012)

12. H. Ramaglia, The flipped mathematics classroom: A mixed methods study examining achievement, active learning, and perception, $\mathrm{PhD}$ Thesis, Kansas State University, (2015)

13. S. Bridoux, Classe inversée pour introduire les suites numériques : une expérience en première année universitaire. In EMF 2018 Paris, France. (2018)

14. A. Sfard, Thinking as communicating: Human development, the growth of discourses, and mathematizing. Cambridge University Press, (2008)

15. A. Sfard, Introduction: Developing mathematical discourse-Some insights from communicational research. Elsevier, (2012)

16. M. Tabach, T. Nachlieli, Combining theories to analyse classroom discourse: A method to study learning process, in Proceedings of the Seventh Congress of the European Society for Research in Mathematics Education, p. 2524-2538, (2011)

17. I. Lavie, A. Steiner, A. Sfard, Routines we live by: from ritual to exploration, ESM, 101, $\mathrm{n}^{\circ} 2$, p. 153-176, (2019), doi: 10.1007/s10649-018-9817-4. 
18. I. Lavie, A. Sfard, How Children Individualize Numerical Routines: Elements of a Discursive Theory in Making, JLS, 28, $\mathrm{n}^{\circ}$ 4-5, p. 419-461, (2019), doi: 10.1080/10508406.2019.1646650.

19. F. Khalloufi-Mouha, Analyse discursive de l'enseignement des fonctions trigonométriques dans la transition lycée/université. in Thomas Hausberger, Marianna Bosch, Faïza Chellougui. Proceedings of the Third Conference of the International Network for Didactic Research in University Mathematics. Sep 2020, Bizerte, Tunisia. University of Carthage and INDRUM, 2020. 〈hal-03084024〉, (2020) 